\title{
Hygienekontrolle von Betriebs- und Spülwasser in Kellereien mittels Kikkoman Lumitester PD-20 und ATP-Teststäbchen
}

\author{
Karin Mandl ${ }^{1}$, Joschy Hummer ${ }^{2}$, Rainer Klinger ${ }^{3}$, und Christoph Mutz ${ }^{1}$ \\ ${ }^{1}$ HBLA und BA für Wein- und Obstbau, Wienerstraße 74, 3400 Klosterneuburg, Austria \\ 2 Kellereinberatung Hummer GmbH, Baumgarten am Wagram 7, 3701 Großweikersdorf, Austria \\ ${ }^{3}$ Biomedica Medizinprodukte GmbH \& Co KG, Divischgasse 4, 1210 Wien, Austria
}

\begin{abstract}
In this experiment RLU (relative light units) values of water of the filling stations of six wineries were examined before and after cleaning. For measurements of the ATP amounts an instrument from Kikkoman was used. This technique is very well suited for cleaning inspection. It shows quickly if the surface of filling station or water is clean or dirty. In this experiment rinse water of six wineries has been tested with LuciPac Pen (cotton swab for surface inspection) and LuciPac Pen-Aqua (special pens for water analysis). For most of the samples, the ATP measurements were made immediately after sample collection, with the remaining samples, the RLU values were taken only in the laboratory. The average ATP values of tap waters are approximately 20 RLU in Austria. This value agrees well with the water value of the Education and Research Center of Viticulture and Pomology in Klosterneuburg. The RLU amount was around 15 RLU. In wineries where well water was used as process water, dangerous germs were isolated. These bacteria were identified by Vitek 2 compact as soil bacteria of the family Pseudomonaceae.
\end{abstract}

\section{Einleitung}

Trinkwasser wird seit vielen Jahren mittels Platten auf Keimbildung (Trinkwasserverordnung, 2001, Satory et al., 2004, WHO, 2006) untersucht. In letzter Zeit setzten sich immer mehr Schnellmethoden wie fluoreszenzmikroskopische Methoden, Flowcytometer und ATP (Adenosintriphosphat) Messungen durch (RintaKanot et al., 2004, Hammes et al., 2008). ATP ist eine wichtige Indikatorsubstanz für Lebendiges im Wasser und auf Oberflächen (Siebel et al., 2008, Venkateswaran et al. 2003). Diese chemische Substanz kann die Zelle verlassen und als Indikator für die Sauberkeit herangezogen werden. Der Ausgangspunkt für die Entwicklung des in diesem Projekt verwendeten Gerätes Kikkomann PD 20 und dessen erste Einsätze waren Großküchen für die ATP Messung. Die Geräte wurden weiter entwickelt für Oberflächenscreening und Wasserkontrollen. Sauberkeit in Betrieben ist sehr wichtig, um Verunreinigungen und Mikroorganismen nicht von einem Lebensmittel auf ein anderes zu übertragen. Die Folgen liegen im Verderb, Senkung der Qualität und Stabilität der Produkte. Es kann zu einem Schweben von Partikeln in Flüssigkeiten kommen, die sich als ungewollte Trübungen oder Biofilme manifestieren. Schnell kommt es $\mathrm{zu}$ Biofilmbildung in den Rohrleitungen (Niquette et al 2000, Hallam et al., 2001, Storgårds et al., 2005), wenn diese nicht angemessen gereinigt werden. Von den Trinkwasserleitungen ist bekannt, dass auf unterschiedlichen Materialen das Wachstum der Bakterien und Biofilmbildung verschieden stark ausgeprägt ist Stehzeiten von Wasser führen zu einer erhöhten ATP Freisetzung, einer Erhöhung der
Bakterienanzahl (Lautenschlager et al., 2010, Carrick et al., 2001), Schwermetallen wie Blei, Nickel, Cadmium und Kupfer (Zietz et al., 2007, 2003) und zusätzlich wirken sich die Stehzeiten negativ auf den Geschmack, den Geruch und die Farbe des Wassers (Servais et al, 1995) aus. Im Verteilungsnetz des Leitungswassers versucht man das bakterielle Wachstum durch niedrige Nährstoffkonzentrationen, Desinfektionsmittel, kurze Stehzeiten und kühle Temperaturen (Servais et al., 1992, Kerneys et al., 1995) ein zu schränken. Bakterien aus dem Wasser und pathogene Keime spielen im Wein nur eine untergeordnete Rolle. Pathogene Keime wie E.coli, Salmonellen und Staphylokokkus aureus zeigen kein Wachstum im Wein. Standzeiten des Weines von 30 min führen bereits zu einem Absterben dieser Bakterien (Ziegelwagner et.al, 2012). Zu bedenken ist, dass viele Keime mehrere Monate auf trockenen Oberflächen (Kramer et al., 2006) überleben und das sollte bei der Wartung und Reinigung von Füllanlagen (Timke et al., 2008, Storgårds et al, 2005) berücksichtigt werden. Füllanlagen neigen bei schlechter Pflege zu starken Biofilmbildungen, die sich negativ auf das Produkt Wein in Form von Qualitätsminderung und Trübungen auswirken. Die Biofilme bilden sich wenige Stunden nach der Reinigung des Stahls wieder und deshalb sollten die Reinigung der Anlage täglich oder unmittelbar vor dem Einsatz erfolgen (Storgårds et al., 2005).

In diesem Projekt wurden verschiedene Wasserqualitäten und Reinigungsverfahren mittels ATP Messungen beschrieben. Anhand von Spülwasserproben wurden die Ergebnisse der Desinfektions- und Waschmaßnahmen (Bodenschatz et al., 2005) erhoben. Es war vor allem 
von Interesse, ob diese ATP Schnellmethode für den praktischen Einsatz im Weinbaubetrieb oder in der Fruchtsaftabfüllung geeignet ist.

\section{Material und methode}

Die Überprüfung der Sauberkeit des Wassers erfolgt in diesem Projekt mit einem Lumitester PD-20 der Firma Kikkoman, Düsseldorf. Das Gerät arbeitet mit einer patentierten ATP/AMP (Adenosinmonophosphat) Luciferastechnik. Dieses Testsystem nutzt eine zyklische Methode basierend auf einer Kombination von Glühwürmchen- Luciferase und Pyruvat Orthophosphatkinase (PPDK) aus. Die Methode produziert eine definierte Menge an Lumineszenz proportional zur Menge des Adenonsintriphosphats (ATP) und Adenosinmonophosphats (AMP) in der Probe und beginnt entsprechend des ATP Gehaltes zu leuchten. Es wurden zwei verschiedene ATP Stäbchen (LuciPac Pen-Aqua und LuciPac Pen verwendet. Die ATP Werte wurden dann in RLU (Relative Light Unit) angegeben.

\subsection{Betriebswasseruntersuchung}

Probennahme Betriebswasser und Spülwasser.

Die Spülwasserproben wurden vor und nach dem Reinigen und anschließend nach der Füllung genommen. Die Ablässe wurden abgeflammt und nach kurzem Laufen des Spülwassers wurden die Proben gezogen. Betriebswasserproben wurden so genommen, dass der Hahn vorher abgeflammt wurde und nach fünf minütigem Vorlauf die Wasserprobe entnommen wurde. Jede Probe wurde zweimal gezogen. Die Proben wurden gekühlt ins Labor transportiert.

\subsection{Betriebe}

Es wurden sechs repräsentative Betriebe mit Nirostafüllanlagen und unterschiedlichen Reinigungsgewohnheiten im Weinviertel ausgewählt.

Der Betrieb A hängt am öffentlichen Leitungswassernetz. Das Wasser wird nach Eintritt in den Betrieb enthärtet und über eine UV -Lampe geführt. Die Reinigung der Anlage erfolgt regelmäßig chemisch mit anschließender Dampfsterilisation. Die Flaschen werden vor Füllung mit ozonisiertem Wasser ausgespült. Die ATP Betriebswasserwerte sind um die 23 RLU und liegen im Normalbereich.

Der Betrieb B wird vom hauseigenen Brunnen mit Wasser versorgt. Das Wasser wird ohne weitere Behandlung als Waschwasser verwendet. Die Füllanlage wird sporadisch mit Chemie und /oder Dampf gereinigt. Die Flaschen werden prinzipiell mit SO2-Lösung ausgespült.

Der Betrieb C ist Fruchtsaftabfüller mit sehr genauer Reinigungspraxis. Zuerst wird mit chlorfreier Lauge bis $85^{\circ} \mathrm{C}$ gereinigt, anschließend wird Wasserstoffperoxid eingesetzt und am Schluss mit Betriebswasser gespült. Das Betriebswasser stammt aus dem öffentlichen Netz, wird enthärtet und über eine UV-Lampe geführt. Alle Produkte werden zusätzlich Hochkurzzeit-erhitzt. Die Werte der Betriebswasserleitung liegen bei 25RLU.
Der Betrieb D führt sporadische chemische Reinigungen der Abfüllanlage durch, hauptsächlich wird die Anlage mit sehr großen Wassermengen aus dem öffentlichen Netz gespeist. Das Betriebswasser wird vorher über eine UVLampe geführt. Das Wasser weist sehr gute Qualität auf die RLU Werte liegen bei 6.

Der Betrieb E ist ein älterer Betrieb, der mit einem hauseigenen Brunnen versorgt wird. Die Reinigung der Abfüllanlage erfolgt teilweise mit Lauge und Wasserstoffperoxid. Angebrannte Belege werden nicht durch die Dampfsterilisation aus den Leitungen und dem Füller aktiv entfernt und lösen sich teilweise sporadisch selbstständig. Die Flaschen werden über Tauchsterilisator mit Peressigsäure geführt, das Nachspülwasser nach dem Sterilisator wird über einen kleinen Kerzenfilter geführt. Die ATP Werte des Betriebes liegen bei über 500RLU Werte im Labor gemessen.

Der Betrieb F reinigt ihre Füllanlage regelmäßig mit Lauge und Wasserstoffperoxid. Das Betriebswasser wird unbehandelt aus eigenem Hausbrunnen verwendet. Die Sterilisation der Anlage erfolgt über Dampf. Das Brunnenwasser weist einen Wert von über 167 RLU Wert im Labor auf.

Die Proben wurden verdünnt und auf verschiedenen Nährmedien ausplattiert und fünf Tage bei $22^{\circ} \mathrm{C}$ bebrütet und danach ausgezählt. Die Proben wurden nicht entsprechend der österreichischen Trinkwasserverordnung (Trinkwasserverordnung, 2001) bearbeitet, da ein Vergleich mit offiziellen Untersuchungswerten nicht beabsichtigt wurde und nicht Ziel der Arbeit war. Es wurde die Hausmethode mit $100 \mu$ l Ausplattieren der Probenflüssigkeit vorgezogen.

\subsection{Nachweis der gefundenen Keime aus den Betrieben}

Die Untersuchung der gefundenen Keime erfolgte durch Reinkultivierung der Bakterien. Die Gramfärbung wurde vor der VITEK@ 2 Compact (BioMérieux, Deutschland) Untersuchung durchgeführt. Anschließend wurden die gefärbten Präparate nochmals nach der klassischen Methode mit den Ergebnissen visuell überprüft.

\section{Ergebnisse und diskussion}

In Tabelle 1 sind die Ergebnisse der RLU Werte von den verschiedenen Wassern dargestellt. Die RLU Werte des Wassers hängen sehr stark mit den Ausgangswerten des Basiswassers zusammen. Diese sollten immer vorher erhoben werden, um einen Bezug zu erhalten. Diese Technik ist sehr gut für den Nachweis von Reinigungen geeignet. Es kann der Status vor und nach der Reinigung einfach mittels eines Stäbchens überprüft werden. Für Fruchtsäfte, die sehr viel ATP enthalten ist das Gerät nicht geeignet. Es hat sich aus den Arbeiten gezeigt, dass sie Testungen mittels Stäbchen unmittelbar nach der Probennahme erfolgen sollte. Standzeiten der Probe oder des $\mathrm{zu}$ untersuchenden Wassers führen $\mathrm{zu}$ drastischen Erhöhungen der ATP Werte wie es bereits in den Publikationen Lautenschlager et al., 2010 und Carrick et al., 2001 beschrieben ist. Mittels eines kleinen Laborantestversuches mittels Hitzeeinwirkung konnte gezeigt 
Tabelle 1. Stellt die Mittelwerte der ATP Messungen(Angabe in RLU) und Keimzahlbestimmungen (Angabe in KBE/ml) der Betriebe A -F dar, - bedeutet, dass keine RLU Werte direkt nach der Probennahme zur Verfügung stehen. PC steht für Plate Count.

\begin{tabular}{|c|c|c|c|c|c|c|}
\hline Betriebe & $\begin{array}{l}\text { Qualität des } \\
\text { Wassers }\end{array}$ & $\begin{array}{l}\text { LuciPacPen } \\
\text { im Keller } \\
\text { RLU }\end{array}$ & $\begin{array}{l}\text { LuciPacPen } \\
\text { im Labor } \\
\text { RLU }\end{array}$ & $\begin{array}{l}\text { LuciPacPen } \\
\text { Aqua im } \\
\text { Keller RLU }\end{array}$ & $\begin{array}{l}\text { LuciPacPenAqua } \\
\text { im Labor RLU }\end{array}$ & $\begin{array}{l}\mathrm{PC} \\
\mathrm{KBE} / \mathrm{ml}\end{array}$ \\
\hline \multirow[t]{3}{*}{ Betrieb A } & Betriebswasser & 23 & 40 & 3 & 36 & 45 \\
\hline & $\begin{array}{l}\text { Nachspülwasser } \\
\text { aus Füller }\end{array}$ & 696 & 890 & 1177 & 1594 & 1870 \\
\hline & $\begin{array}{l}\text { Nachspülwasser } \\
\text { aus Füller nach } \\
\text { der Füllung }\end{array}$ & 1840 & 2665 & 1786 & 3047 & 2670 \\
\hline \multirow[t]{3}{*}{ Betrieb B } & Betriebswasser & - & 88 & - & 117 & 1330 \\
\hline & $\begin{array}{l}\text { Nachspülwasser } \\
\text { vor Reinigung }\end{array}$ & - & 526 & - & 526 & 145000 \\
\hline & $\begin{array}{l}\text { Nachspülwasser } \\
\text { nach Abfüllung }\end{array}$ & - & 3013 & - & 8239 & 816000 \\
\hline \multirow[t]{3}{*}{ Betrieb C } & Betriebswasser & 25 & 45 & 26 & 25 & 52 \\
\hline & $\begin{array}{l}\text { Nachspülwasser } \\
\text { aus Füller }\end{array}$ & 9 & 19 & 22 & 10 & 0 \\
\hline & $\begin{array}{l}\text { Nachspülwasser } \\
\text { nach Füllung }\end{array}$ & 10 & 21 & 6 & 13 & 495 \\
\hline \multirow[t]{3}{*}{ Betrieb D } & Betriebswasser & 6 & 9 & 0 & 4 & 0 \\
\hline & $\begin{array}{l}\text { Nachspülwasser } \\
\text { nach Dampfs- } \\
\text { terilisation }\end{array}$ & 2 & 7 & 1 & 2 & 5 \\
\hline & $\begin{array}{lr}\text { Füllorgan } & 1 . \\
\text { Spülung } & \text { nach } \\
\text { Füllung } & \\
\end{array}$ & 8 & 20 & 8 & 5 & 25 \\
\hline \multirow[t]{3}{*}{ Betrieb E } & Betriebswasser & - & 660 & - & 553 & 184000 \\
\hline & $\begin{array}{ll}\text { Füllorgan } & \text { vor } \\
\text { Reinigung } & \end{array}$ & - & 1319 & - & 4825 & 26400000 \\
\hline & $\begin{array}{l}\text { Füllorgan nach } \\
\text { Reinigung }\end{array}$ & - & 231 & - & 208 & 46400 \\
\hline \multirow[t]{3}{*}{ Betrieb F } & Betriebswasser & 31 & 167 & 12 & 238 & 441 \\
\hline & $\begin{array}{ll}\text { Füllorgan } & \text { vor } \\
\text { Reinigung } & \end{array}$ & 30 & 670 & - & 735 & 1980000 \\
\hline & $\begin{array}{l}\text { Füllorgan nach } \\
\text { Reinigung }\end{array}$ & - & 590 & - & 450 & 362000 \\
\hline
\end{tabular}

werden, dass durch die Denaturierung der Zellmembran, es zu einem erhöhten Austritt von ATP kommt. Da lebendige Zellen immer der Autolyse unterliegen, kommt es dadurch zu kontinuierlichen vermehrten Austritt von ATP. Beobachtungen während den Untersuchungen zeigten, dass es lokal in den Flüssigkeiten zu erhöhten Werten führen kann. Deshalb sollte bei Ausreißern unbedingt eine Wiederholung der Messung erfolgen, da das ATP in diesem Bereich nicht homogen verteilt ist. Die Werte selbst sind mittels des Gerätes gut reproduzierbar.

In diesem hier vorgestellten Versuch wurde 6 Kellereien auf Nachspülwasser und Betriebswasser mittels ATP Stäbchen (LuciPac Pen-Aqua und LuciPac Pen) untersucht. Bei einigen Proben wurden die ATP Messungen unmittelbar nach der Probenziehung gemessen. Zu diesem Zeitpunkt war uns noch nicht bekannt, dass die ATP Werte durch die Standzeiten stark ansteigen (Lautenschlager et al., 2010, Carrick et al., 2001), und bei den restlichen Proben wurden die RLU Werte erst im Labor erhoben. Das Betriebswasser der meisten Betriebe lag bei ca. 20 RLU, dies entspricht den durchschnittlichen RLU Werten von Leitungswasser. Diese Werte stimmen auch mit den Wasserwerten des eigenen Labors in Klosterneuburg überein, die Wasserleitungswerte im Labor lagen um die 15 RLU. Es ist interessant indirekt über das Spülwasser des Füllers fest zu stellen, ob die Reinigung zufriedenstellend durchgeführt wurde oder wiederholt gehört. Im Labor wurden von jeder Probe die RLU Werte genommen und die Keime wurden auf Plate Count Medium ausplattiert.

Das Weingut A hängt am öffentlichen Leitungswassernetz. Das Wasser wird nach Eintritt in den Betrieb enthärtet und über eine UV -Lampe geführt. Die Reinigung der Anlage erfolgt regelmäßig chemisch mit anschließender Dampfsterilisation. Die Flaschen werden vor Füllung mit ozonisiertem Wasser ausgespült. Die ATP Betriebswasserwerte sind um die 23 RLU und liegen im Normalbereich. Das Spülwasser ATP Wert liegt nach der Dampfsterilisation liegt bei 696 RLU und ist hoch und spiegelt sich in der Gesamtkeimzahl von $1870 \mathrm{KBE} / \mathrm{ml}$ wieder. Dieser hohe Keimgehalt ist als mikrobiologisch bedenklich zu bewerten. Die Messungen nach der Füllung haben noch höhere Werte ergeben, die sich sowohl in 
den ATP Werten wie in den Keimzahlen des Spülwassers zeigen. In diesem Fall wäre es von Vorteil längere und gründlichere Spülungen mit Wasser durchzuführen. Alleine das würde bewirken, dass die RLU Werte gesenkt werden könnten.

Das Weingut B wird vom hauseigenen Brunnen mit Wasser versorgt. Das Wasser wird ohne weitere Behandlung als Waschwasser verwendet. Die Füllanlage wird sporadisch mit Chemie und /oder Dampf gereinigt. Die Flaschen werden prinzipiell mit SO2-Lösung ausgespült. Das Betriebswasser mit dem ATP- Wert 88 RLU im Labor ist hoch und spiegelt sich in einer Keimzahl von $1330 \mathrm{KBE} / \mathrm{ml}$ wieder. Hier muss darauf hingewiesen werden, dass die Werte erst nach einer gewissen Standzeit erhoben wurden und dies zu einer Erhöhung der RLU Werte führte. Das Nachspülwasser nach der Dampfsterilisation weist ATP Werte von über 500 auf mit einer Keimzahl von mehr als $1,45 \times 10^{5}$ Keimen auf. Nach der Abfüllung stieg der ATP Wert auf über 3000RLU an und die Keimzahl wies 8, $1 \times 10^{5}$ Keime auf. In diesem Betrieb ist das mikrobiologische Risiko der Verschleppung der Keime sehr hoch. In diesem Fall könnte das Wasser aus dem öffentlichen Netz bezogen oder eine UV Lampe vorgeschaltet werden, um Verbesserungen der RLU Werte und eine erhöhte Produktsicherheit zu erreichen.

Das Weingut $\mathrm{C}$ ist Fruchtsaftabfüller mit sehr genauer Reinigungspraxis. Zuerst wird mit chlorfreier Lauge bis $85^{\circ} \mathrm{C}$ gereinigt, anschließend wird Wasserstoffperoxid eingesetzt und am Schluss mit Betriebswasser gespült. Das Betriebswasser stammt aus dem öffentlichen Netz, wird enthärtet und über eine UV-Lampe geführt. Alle Produkte werden zusätzlich Hochkurzzeit-erhitzt. Die Werte der Betriebswasserleitung liegen bei 25RLU. Nach der Dampfsterilisation zeigt das Wasser sehr geringe ATP Werte und die Keimzahl ist auf Null gesunken. Nach der Füllung konnte ein RLU Wert von 10 gemessen werden, der sehr gut ist.

Das Weingut D führt sporadische chemische Reinigungen der Abfüllanlage durch, hauptsächlich wird die Anlage mit sehr großen Wassermengen aus dem öffentlichen Netz gespeist. Das Betriebswasser wird vorher über eine UV-Lampe geführt. Das Wasser weist sehr gute Qualität auf die RLU Werte liegen bei 6 und zeigt sich in der Keimzahl $0 \mathrm{KBE} / \mathrm{ml}$. Nach der Dampfsterilisation fallen die RLU Werte sogar auf 2 ab. Nach der Füllung steigt der Wert wieder auf 8 an. Diese Werte sind optimal aus mikrobiologischer Sicht. Dieser Betrieb hat durch die sehr gute Wasserqualität und das ausgezeichnete Hygienemanagement das mikrobiologische Infektionsrisiko für ihre Produkte stark eingedämmt.

Das Weingut E ist ein älterer Betrieb, der mit einem hauseigenen Brunnen versorgt wird. Die Reinigung der Abfüllanlage erfolgt teilweise mit Lauge und Wasserstoffperoxid. Angebrannte Belege werden nicht durch die Dampfsterilisation aus den Leitungen und dem Füller aktiv entfernt und lösen sich teilweise sporadisch selbstständig. Die Flaschen werden über Tauchsterilisator mit Peressigsäure geführt, das Nachspülwasser nach dem Sterilisator wird über einen kleinen Kerzenfilter geführt. Die ATP Werte des Betriebes liegen bei über 500RLU
Werte im Labor (hier gab es Standzeiten der Proben, die zu einer Erhöhung der Werte führte) und die Keimzahl ist entsprechend hoch bei $1,8 \times 10^{5} \mathrm{KBE} / \mathrm{ml}$. Hier könnten Verbesserungen erreicht werden durch eine UV-Lampe oder dem Anschluss an das öffentliche Netz.

Das Weingut $F$ reinigt ihre Füllanlage regelmäßig mit Lauge und Wasserstoffperoxid. Das Betriebswasser wird unbehandelt aus eigenem Hausbrunnen verwendet. Die Sterilisation der Anlage erfolgt über Dampf. Das Brunnenwasser weist einen Wert von über 167 RLU Wert im Labor (hier gab es Standzeiten der Proben, die zu einer Erhöhung der Werte führte) auf und die Keimzahl ist bei $441 \mathrm{KBE} / \mathrm{ml}$ Wasser. Die ATP Werte liegen vor der Reinigung bei 670 mit einer Keimzahl von 1,9 $\times$ $10^{6} \mathrm{KBE} / \mathrm{ml}$ Spülwasser. Nach der Reinigung sinken die ATP Werte auf 590 ATP Werte und die Keime auf $3,6 \times 105 \mathrm{KBE} / \mathrm{ml}$ Spülwasser. Die Keimzahlen stellen trotz Berücksichtigung der Standzeiten ein mikrobiologisches Risiko für das Produkt dar.

\section{Gefundene wasserkeime}

In den Betrieben mit eigener Versorgung von Hausbrunnen als Waschwasser konnten bedenkliche Keime isoliert werden. Diese Keime wurden mit Vitek compakt 2 identifiziert. Es handelt sich hier vor allem um Bodenkeime der Familie Pseudomonaceae.

\section{Literaturhinweise}

S. Bodenschatz, Kompaktwissen Desinfektion: Neuauflage des Standardwerks, Handbuch für den Desinfektor, Behr's Verlag (2005)

K. Carrick., M. Barney, A. Navarro, D. Ryder, The comparison of four bioluminometer and their swab kits for instant hygiene monitoring and detection of microorganisms in the brewery, Journal of Institute of Brewing, 107, Nr. 1 (2001)

N.B, Hallam, J.R. West, C.F. Forster, and J. Simms, The potential for biofilm growth in water distribution systems, water Res. 35, 17, 4063-4071 (2001)

F. Hammes, M. Berney, Y. Wang, M. Vital, O. Köster and T. Egli, Flow- Cytometric total bacterial cell counts as a descriptive microbiological parameter for drinking water treatment processes, water Res., 42 (1-2), 269-277 (2008)

A. Kramer, I. Schwebke und G. Kampf, How long do nosocomial pathogens persit on inanimate surfaces? A systematic review BMC Infectious Diseaeses, 6, 130 (2006)

A. Kerneys, F. Nakache, A. Deguin, M. Feinberg,. The effects of water residence time on the biological quality in a distribution network. Water Res. 29, 1719-1727 (1995)

K. Lautenschlager, N. Boon Y. Wang, T. Egli., F. Hammes, Overnight stagnation of drinking water in household taps induces microbial growth and changes in community composition Water Research 44, 2010, 4868-4877

P. Niquette, P. Servais, R. Savoir,. Impact of pipe materials on densities of fixed bacterial biomass in a drinking water distribution system. Water Res. 34, 1952-1956 (2000) 
J.M. Rinta - Kanto, M.J. Lehtola, T. Vartianinen and P.J. Martikainen. Rapid enumeration of virus - like particles in drinking water samples using SYBR green I-Staining, Water Res., 38, 2614-2618 (2004)

D.P. Satory, Heterotrophic plate count monitoring of treated drinking water in the UK, a useful operation tool, Int. J. Food Microbiol., 92, 297-306 (2004)

P. Servais, G. Billen, P. Laurent, Y. Levi, G. Randon, Studies of BDOC and bacterial dynamics in the drinking water distribution system of the Northern Parisian suburbs. Revue das Sci. de léau 5, 69-89 (1992)

P. Servais, P. Laurent and G. Randon Comparison of bacterial dynamics in various French distribution systems J. Water Sci. Res. Technol. -Aqua 44, 10-17 (1995)

E. Siebel, Y. Wang, T. Egli, and F. Hammes Correlation between total cell concentration, total adenosine triphosphate concentration and heterotrophic plate counts during microbial monitoring of drinking water, Drinking Engeneering and Science Discussions 1, 71-86 (2008)

E. Storgårds, K. Tapani, O. Sinebrychoff, P. Hartwall, O. Hartwall, R. Saleva, O. Oyj, and M-L. Suihko, J. Microbial Attachment and Biofilm Formation in Brewery Bottling Plants. Am. Soc. Brew. Chem. 64(1), 8-15 (2006)

M. Timke, Wang-Lieu, K. Altendorfer, A. Lipski, Identity, beer spoiling and biofilm forming potential of yeasts from beer bottling plant associated biofilms, Antonie van Leeuwenhoek 93, 151-161 (2008)

Trinkwasserverordnung Verordnung des Bundesministers für soziale Sicherheit und Generationen über die Qualität von Wasser für den menschlichen Gebrauch (Trinkwasserverordnung - TWV) StF: BGB1. II Nr. 304/2001 [CELEX-Nr.: 398L0083] (2001)

C. Ziegelwagner, K. Silhavy-Richter, und K. Mandl, Antimikrobielle Wirkung von Wein Ithakajournal, 1, 4756 (2012)

B.P. Zietz, J.D. de Vergara, H. Dunkelberg Copper concentrations in tap water and possible effects on infant 's health results of a study in Lower Saxony, Germany, Environ. Res. 92, 129-138 (2003)

B.P Zietz,. J. Lass, R. Suchenwirth Assessment and management of tap water lead concetration in Lower Saxony, Germany. Int. J. Environ. Health Res. 17, 407418 (2007)

K. Venkateswaran, N. Hattori, L.D. Myron, R. Kern ATP as a biomarker of viable microorganisms in clean-room facilities Journal of Microbiological Methods 52, 367-377 (2003)

WHO: World Health Organization, Guide lines for Drinking-water Quality (electronic resource): incorporating first addendum, 1, Recommendations, 3.Ed., (2006) 